\title{
ETAPAS DEL SARLAFT
}

Identificación del riesgo: si se hace en atención al volumen de actividades, cantidad de clientes y productos ofrecidos, será mucho más fácil identificar los segmentos de cada uno de los factores de riesgo. Para segmentar los diferentes factores se recomienda tener en cuenta los criterios establecidos en el Título I, Capítulo XI de la Circular Externa 007 de 1996 (Circular Básica Jurídica), de la Superintendencia Financiera y la división natural de cada uno de ellos.

Los eventos de riesgo son el resultado de revisar cada segmento y la incidencia posible del riesgo de LA/FT de manera permanente, teniendo en cuenta que los eventos corresponden a hechos ocurridos efectivamente desde la creación de la compañía.

× Medición de la probabilidad y el impacto del riesgo de LA/FT.

× Medición o evaluación del impacto que ha de generar la ocurrencia del riesgo.

$\times$ El control del riesgo de lavado de activos y financiación del terrorismo a implementar.

$\times$ El monitoreo del riesgo de lavado de activos y financiación del terrorismo con la periodicidad oportuna, de acuerdo con las necesidades. 
Figura 2.1. Etapas del SARLAFT

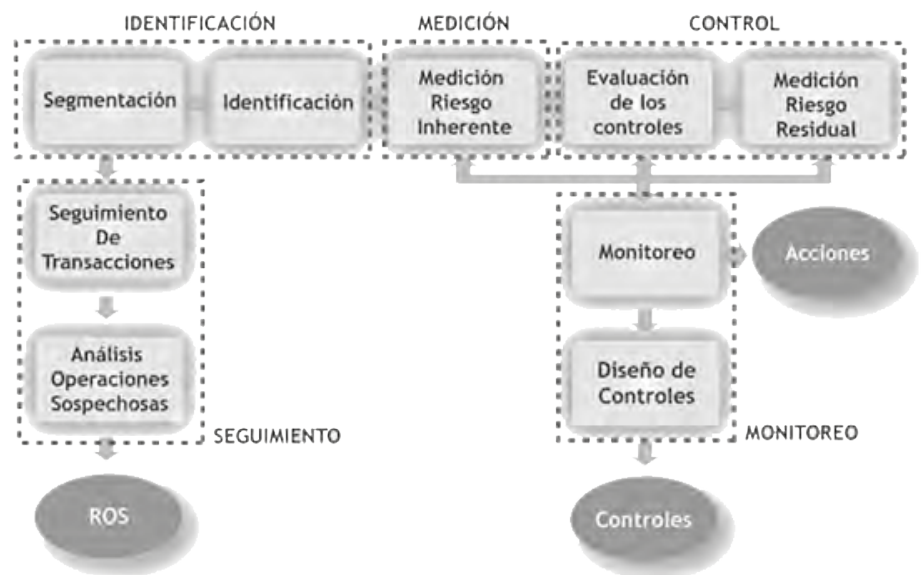

Fuente: elaboración propia

\subsection{Metodología para la identificación del riesgo}

Para la identificación del riesgo existen varias metodologías contenidas en la Norma Técnica Colombiana NTC 5254 de ICONTEC, herramienta principal para establecer los procesos internos en cualquier empresa o entidad, una de ellas a modo de ejemplo es la relacionada en el numeral 3.3:

\subsubsection{Juicios basados en la experiencia y los registros}

× Primero se debe determinar cuáles serán los objetivos en materia de LA/FT.

× Luego se debe identificar cuáles han sido las tipologías de los delitos que más se han reportado en el ejercicio de la actividad que realizan.

* Una vez identificadas las tipologías más comunes se pueden definir cuáles serían las mejores prácticas por realizar para prevenir el LA/FT (es muy útil recopilar información contenida en documentos nacionales e internacionales que contengan asuntos sobre LA/FT).

× Consultar la información y la experiencia interna que se ha tenido en materia de LA/FT en la empresa. 
× Definir la forma (sintaxis), para expresar los eventos de riesgos a los que puede verse expuesta la entidad.

* Elaborar una lista de los eventos de riesgo de LAIFT para la empresa (la cual deberá ir alimentando de forma permanente con las nuevas modalidades de señales de alerta que se identifiquen a futuro).

* Identificar el modelo o metodología de segmentación de los factores de riesgo que más se adecua a las necesidades de la empresa, para con ello hacer ejercicios piloto que den como resultado mediciones de la probabilidad y el impacto del riesgo de LA/FT al que está permanentemente expuesta la entidad, y así ir formulando nuevas políticas y medidas de mitigación.

\subsubsection{Seguimiento de operaciones para el análisis de operaciones sospechosas}

Luego de identificar los riesgos y de segmentar los factores que los generan se puede usar una de las clasificaciones u organización en forma de árbol, similar a un dendrograma, usando las siguientes características para realizar el análisis de las actividades de los clientes o personal interno y externo:

Figura 2.2. caracteristicas para realizar análisis de actividades de clientes

Los clientes o personas se clasifican teniendo en cuenta lis variables que describen en su actividad económica, volumen o frecuencia de transacciones u operaciones, monto de ingresos y egresos y patrimonio.

Los productos se dividen o segmentan temiendo en cuenta su naturaleza, caracteristicas y nicho de mercado o destinatarios.

Los canales de distribución se segmentan teniendo en cuenta su naturaleza y caracteristicas.

Las jurisdicciones se segmentan según la ubicación, caracteristicas y naturaleza de las transacciones 
Otra forma de seguimiento y análisis de las operaciones de los clientes o personal externo e interno en el análisis de los datos obtenidos en la identificación es la estructura la segmentación de los factores de riesgo, mediante la técnica estadística de minería de datos.

La segmentación como técnica estadística tiene mucha relación con la minería de datos, por lo tanto, existen varias metodologías generales para realizar esta labor. Una de las metodologías más reconocida por minería de datos es la KDD, la cual sirve como marco conceptual.

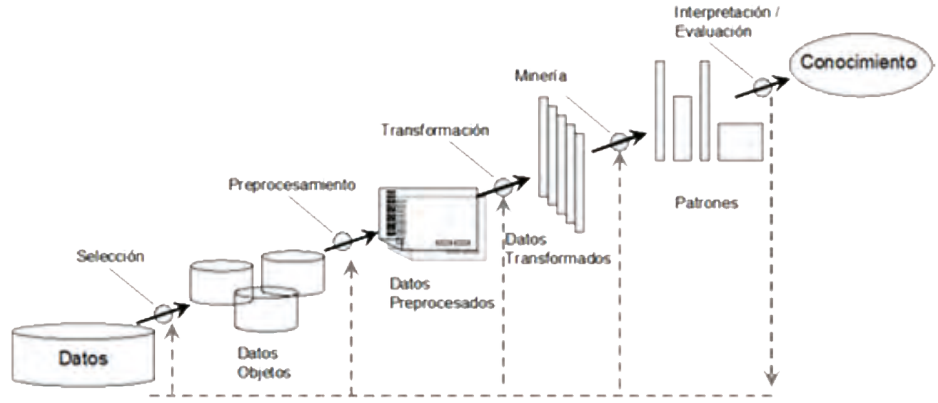

Fuente: Francisco Ibarra - Descubrimiento del conocimiento (KDD) "el proceso de minería".

Figura 2.4. Las etapas del KDD
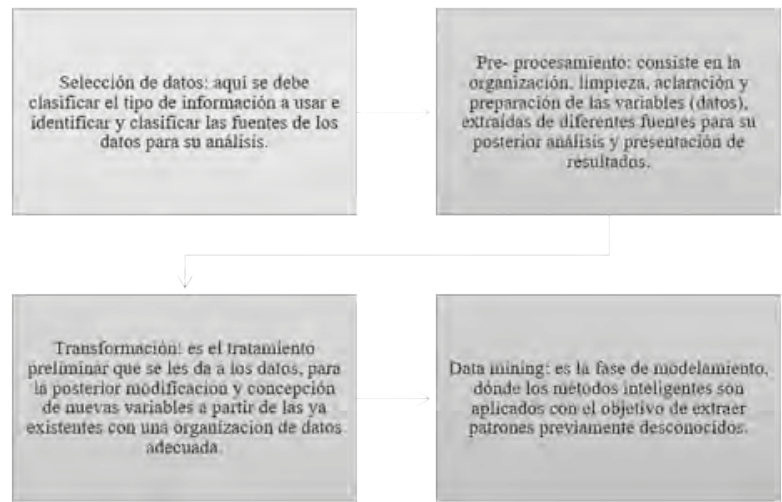

Fuente: elaboración propia 
Una vez definida la metodología de minería de datos, se debe seleccionar la técnica de segmentación más apropiada y el enfoque que esta tiene. Dicho proceso de selección se denomina procedimientos estadísticos de segmentación, y se realiza con el fin de que la segmentación se fundamente no solo en una técnica, sino que tenga en cuenta otros aspectos como el algoritmo, el modelo y el método.

\subsubsection{Los procedimientos estadísticos más empleados}

Figura 2.5. Procedimientos estadísticos más empleados

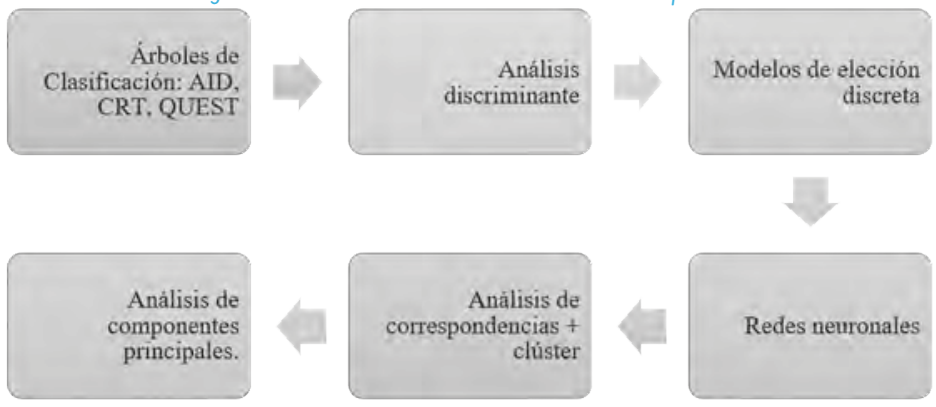

Fuente: elaboración propia

\subsubsection{Las entradas que se necesitan para realizar la seg- mentación}

× Entradas externas: para segmentar jurisdicciones.

$\times$ Entradas internas:

Figura 2.6. Entradas internas.

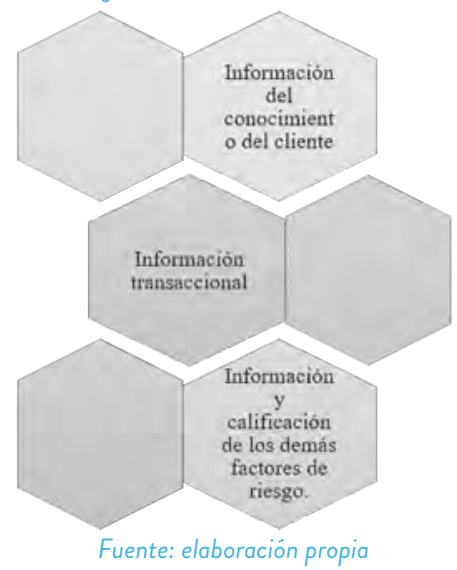




\subsubsection{Proceso de segmentación de los factores de riesgo}

Con base en la información disponible de la entidad o empresa, se seleccionan las bases de datos:

\subsubsection{Segmentación clientes o personal interno y/o externo}

Se trabaja la segmentación de clientes o personal interno o externo con la base completa de estos, en donde se conformarán los primeros conglomerados de la siguiente forma y bajo el siguiente criterio:

Figura 2.7. Segmentación clientes
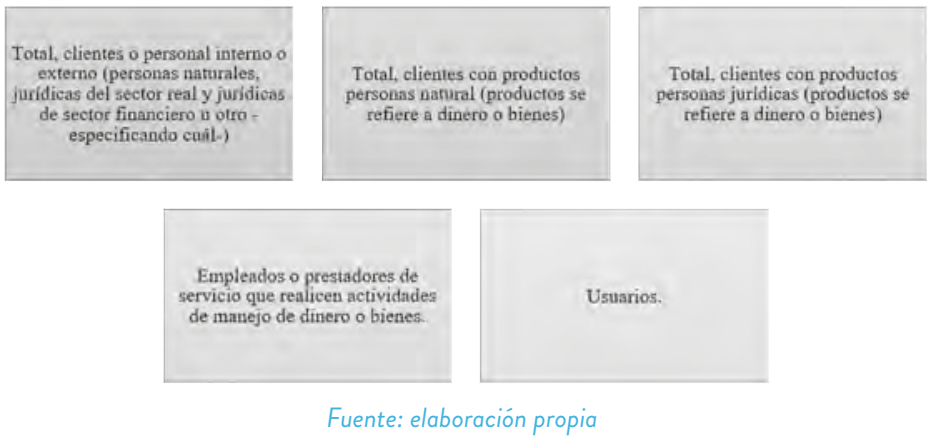

× Segmentación de productos: los productos y servicios que se toman para la segmentación deben dividirse y subdividirse de acuerdo con los tipos de productos o subproductos que tenga.

× Segmentación para los canales de distribución: los canales que se toman para la segmentación se subdividieron de acuerdo con los subtipos de canales que se tienen.

* Segmentación para la jurisdicción: para la segmentación de las jurisdicciones se toman en cuenta todos los departamentos del país, teniendo en cuenta la cantidad de delitos relacionados a LA/FT que reportan cada uno de estos y el tamaño de la población.

Para la segmentación por productos, canales y jurisdicción es de gran ayuda usar los datos emitidos por la UIAF en el informe de resultados consolidados a nivel nacional entre el año 2017 y 2018 del informe de evaluación por regiones producto de encuestas a la población así: 
- Año 2017: los encuestados identificaron como amenazas para el lavado de dineros provenientes de actividades ilicitas -en el orden en que se plasman- el narcotráfico, el contrabando, la extorsión, la evasión tributaria y la minería ilegal, mientras que actividades como el tráfico de armas, la trata de personas, el tráfico de inmigrantes y el tráfico de menores tuvieron porcentajes más bajos en la percepción de la muestra de la encuesta.

* Vulnerabilidad a nivel nacional año 2017: se identificaron como actos vulnerables al lavado de activos, en el orden en que se relacionan, el comercio al por mayor y detal, la construcción de obras civiles, el mercado inmobiliario, la ganadería, la explotación de minas y canteras, hoteles y restaurantes, transporte de pasajeros, producción de textiles, agricultura, caza, silvicultura y pesca, servicios financieros y servicios públicos, productos químicos, servicios de educación y salud, y las actividades de papelería.

* Cumplimiento normativo año 2017: en este punto se preguntó a los encuestados sobre el nivel de cumplimiento normativo en el país para la prevención del lavado de activos y financiación del terrorismo en algunas actividades, de lo cual se obtuvo que para los colombianos las entidades sí incluyen estrategias para luchar contra el LA/FT dentro de sus manuales, cuentan con oficial de cumplimiento, tienen señales de alerta para identificar tipologías penales en materia de LA/FT, realizan capacitación a su personal en materia de lucha contra el LA/FT, hacen mayor control en materia de debida diligencia $^{3}$, en seguimiento a las operaciones de los clientes, en verificación de clientes en lista restrictiva del Consejo de Seguridad de las Naciones Unidas a personas políticamente expuestas (PEP), en la elaboración de mapas de riesgo y en las operaciones con países de alto riesgo.

* Año 2018: el ejercicio de la evaluación dio como resultado que los encuestados ratificaron las principales amenazas del 2017, pero en esta ocasión con porcentajes de percepción más altos, de tal manera que la corrupción paso de un $57 \%$ a un $67 \%$, el narcotráfico de un $46 \%$ a un $58 \%$, el contrabando de un $42 \%$ a un $57 \%$ y la extorsión de un $40 \%$ a un $44 \%$. La figura de la evasión tributaria se incluyó como una nueva fuente de lavado de activos en la encuesta, la cual tuvo un puntaje de 38\%, y la minería ilegal disminuyó su nivel de apreciación, pasando de un 32\% a un 19\%, al igual que el tráfico de armas, la trata de personas, el tráfico de inmigrantes, y el tráfico de menores que estaba entre el $24 \%$ y el $17 \%$ pasó a oscilar entre el $19 \%$ y el $9 \%$.

\footnotetext{
${ }^{2}$ Actividades económicas, canales, servicios, activos y la implementación de controles normativos 
* Vulnerabilidad a nivel nacional año 2018: para 2018 se identificaron como actos vulnerables para la comisión de lavado de activos algunos del año anterior, pero en esta ocasión disminuyendo los porcentajes de percepción e identificando nuevas actividades, de tal manera que el comercio al por mayor y detal paso del $65 \%$ al 37\%, la construcción de obras civiles del 53\% al $31 \%$, el mercado inmobiliario del $52 \%$ al $43 \%$, la ganadería del $53 \%$ al $24 \%$, la explotación de minas y canteras, hoteles y restaurantes del 52\% al 23\%, el transporte de pasajeros y carga del $40 \%$ al 21\%, la producción de textiles del $16 \%$ al $15 \%$, la agricultura, caza, silvicultura y pesca del $26 \%$ al $13 \%$, los servicios financieros y servicios públicos del 19\% al 14\%, los productos químicos del $16 \%$ al $7 \%$, los servicios de educación y salud del $14 \%$ al $7 \%$ y las actividades de papelería del $16 \%$ al $6 \%$; adicionalmente, se identificaron como nuevas fuentes de vulnerabilidad las entidades sin ánimo de lucro con un 25\%, la comercialización de vehículos con un $22 \%$, la comercialización de arte, joyas y bienes de lujo con un $22 \%$, la comercialización de combustibles, empresas de seguridad y vigilancia, actividades deportivas y tala de árboles que oscilan entre el $17 \%$ y el $4 \%$.

Figura 2.8. Proporción de encuestados que perciben ciertas actividades como vulnerables 2017-2018.

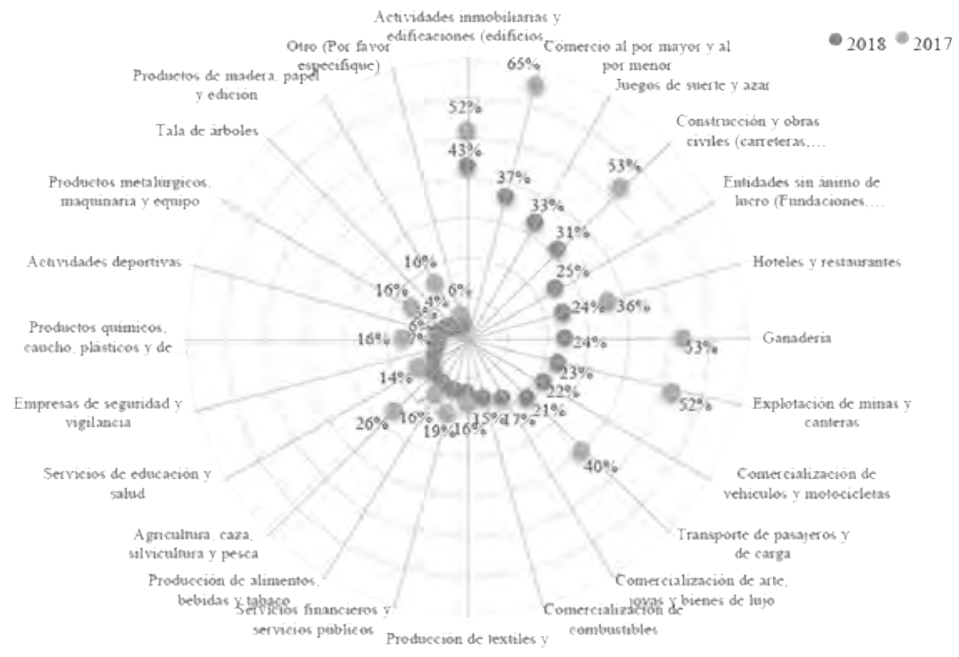

Fuente: elaboración UIAF con base en resultados de la Encuesta de percepción del riesgo de lavado de activos y financiación del terrorismo 2018. 
× Operaciones vulnerables a nivel nacional año 2018: este módulo de vulnerabilidad es nuevo para la evaluación, por lo que la pregunta referente a este tema se incluyó por primera vez dentro de la evaluación 2018; de allí que estos son los primeros datos obtenidos en materia de percepción sobre identificación de operaciones comerciales y financieras que más se usan para la comisión de delitos, de lo cual se obtuvo que para los colombianos estas son el pago en efectivo (billetes y monedas), con un 60\%, el intercambio de bienes (a título gratuito o simulaciones de contratos onerosos), con un 52\%, los giros nacionales en oficinas postales con un $27 \%$, operaciones nacionales en el sistema financiero en efectivo con un $27 \%$, actividades en moneda digital con un $25 \%$, los giros internacionales en efectivo en oficinas postales con un $23 \%$, las transacciones internacionales en efectivo por oficinas postales $17 \%$, y operaciones nacionales en divisas en el sistema financiero con un 17\%.

Figura 2.9. Proporción de percepción operaciones vulnerables a nivel nacional año 2018

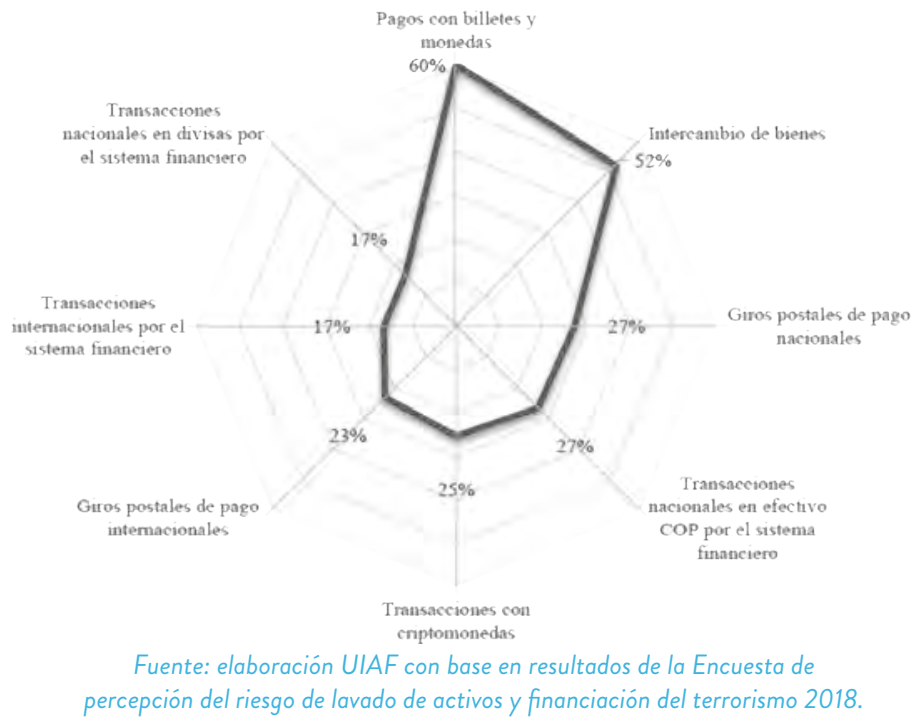

× Servicios vulnerables a nivel nacional 2018: este es el segundo módulo de vulnerabilidad novedoso para el año 2018, de allí que los resultados obtenidos de la apreciación de los colombianos destacan que los servicios más usados por los delincuentes para encubrir sus actividades son las operaciones inmobiliarias con un $50 \%$, la constitución de sociedades con un 37\%, las operaciones contables 
con un 35\%, la compra y venta de divisas con un 34\%, los trámites notariales con un $29 \%$, las zonas francas, aduaneras y puertos marítimos con un $19 \%$, los fideicomisos con un $11 \%$ y el transporte de valores con un $9 \%$.

Figura 2.10. Proporción de percepción de servicios vulnerables a nivel nacional 2018

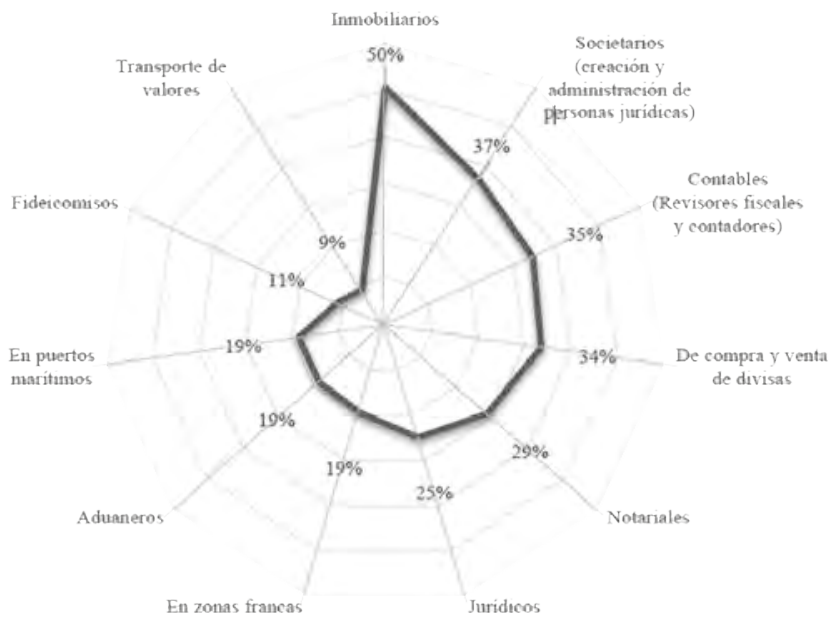

Fuente: elaboración UIAF con base en resultados de la Encuesta de percepción del riesgo de lavado de activos y financiación del terrorismo 2018.

× Activos vulnerables a nivel nacional año 2018: este módulo también es nuevo en el informe de evaluación de vulnerabilidades, por lo que los encuestados manifestaron que los activos más vulnerables para el ocultamiento de dineros ilícitos son los bienes inmuebles urbanos con un 58\%, los bienes inmuebles y terrenos rurales con un $52 \%$, el dinero en efectivo con un $39 \%$, los terrenos urbanos con un $36 \%$, las inversiones en el exterior con un $26 \%$, las operaciones en moneda digital con un $24 \%$, operaciones en divisas con un $21 \%$, las inversiones en el sector real con un $19 \%$, los metales y las piedras preciosas con un $18 \%$, joyas, artículos de lujo e inversiones en sector financiero con un 15\%, las obras de arte, antigüedades y objetos de colección con un 12\% y los animales exóticos y de razas finas con un $11 \%$. 
Figura 2.11. Proporción de percepción de activos vulnerables a nivel nacional 2018

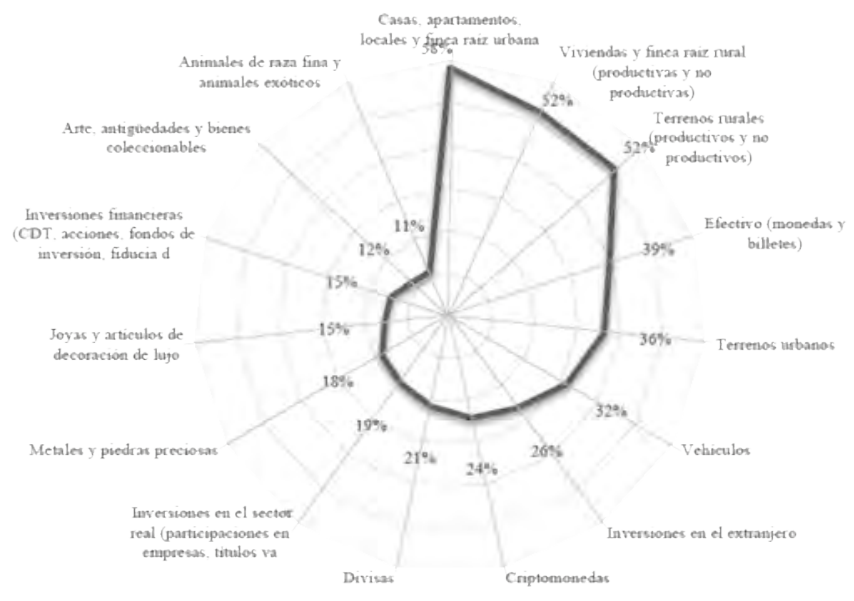

Fuente: elaboración UIAF con base en resultados de la Encuesta de percepción del riesgo de lavado de activos y financiación del terrorismo 2018.

× Cumplimiento normativo 2018: para el año 2018 los encuestados sostuvieron las mismas actividades como las más controladas por las entidades financieras, en esta ocasión con porcentajes más altos; además, se incluyeron nuevas actividades que consideran que también dan cumplimiento a la debida diligencia de conocimiento del cliente, entendida esta como "(...) el conocimiento de hábitos de consumo, preferencias y gustos; pero para efectos de la prevención del lavado de activos, el conocimiento de la persona natural o jurídica debe ser más profundo, ya que implica la recolección de información relevante - como por ejemplo movimientos financieros, operaciones con la empresa y posibles operaciones de tipo inusual, etc. - que permita detectar y analizar operaciones sospechosas (...)" 4.

Las actividades que según los colombianos cumplen con la debida diligencia son la implementación de estrategias para luchar contra el LA/FT dentro de sus manuales, que pasó del $87 \%$ al 91\%; las entidades cuentan con oficial de cumplimiento, que pasó del 75\% al 90\%; tienen identificadas señales de alerta para la identificación de tipologías penales en materia de LA/FT, que pasó del 
$36 \%$ al $87 \%$; realizan capacitación a su personal en materia de lucha contra el LA/FT, que pasó del $71 \%$ al $84 \%$, y hacen mayor control en materia de debida diligencia en seguimiento a las operaciones de los clientes, que pasó del 69\% al $77 \%$.

Para la nueva evaluación 2018 se adicionaron como controles que también se implementan la verificación de clientes en la lista restrictiva del Consejo de Seguridad de las Naciones Unidas a personas políticamente expuestas (PEP), en la elaboración de mapas de riesgo y a las operaciones con países de alto riesgo, que pasó del $47 \%$ al 53\%; las operaciones de los clientes, que pasó de un cumplimiento del $77 \%$ a un $91 \%$; la verificación de clientes en lista restrictiva del Consejo de Seguridad de las Naciones Unidas, que pasó de un $69 \%$ a un $77 \%$. Como actividades nuevas está la conservación de registro de sus clientes del $96 \%$, el monitoreo a personas políticamente expuestas (PEP) con un 53\%, en la elaboración de mapas de riesgo con un $53 \%$ y a las operaciones con países de alto riesgo con el $48 \%$.

Un ejemplo de lo antes planteado son los resultados de los diferentes análisis realizados por entes públicos y privados elaborados en Colombia entre los años 2000 y 2017 , de los cuales se obtuvo que la principal causa del LA/FT y sus delitos subyacentes es la corrupción, así.

Figura 2.12. Proporción de percepción de cumplimiento a implementación de mecanismos de prevención y detección de LA/FT 2017-2018

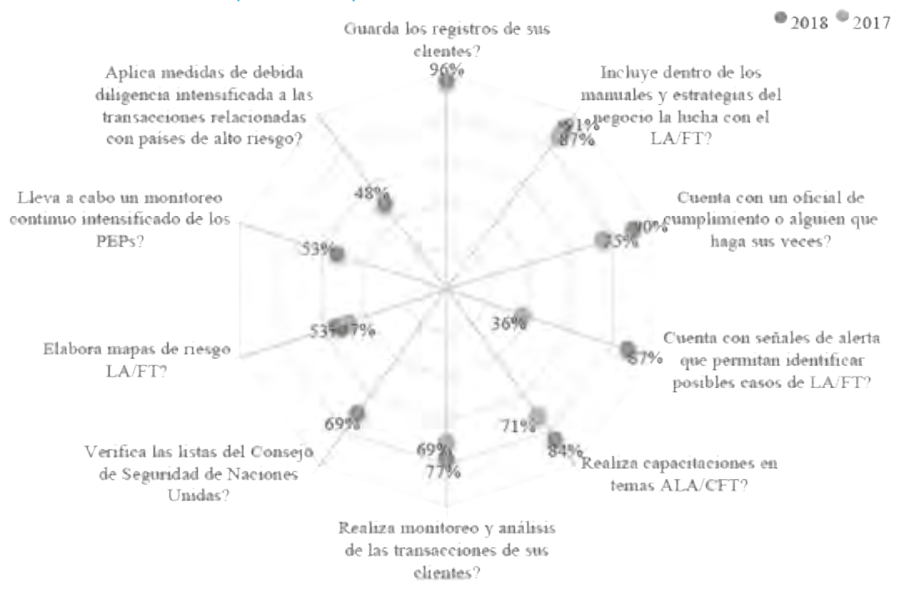

Fuente: elaboración UIAF con base en resultados de la Encuesta de percepción del riesgo de lavado de activos y financiación del terrorismo 2018. 


\subsubsection{Medición o evaluación}

Para la medición del riesgo por primera vez, dentro de una empresa o entidad, una de las metodologías recomendadas a emplear es la:

\subsubsection{Encuesta a expertos}

Este método, contenido en la Norma Técnica Colombiana NTC 5254 del Icontec, se utiliza para la medición de la probabilidad y el impacto, por lo que se fundamenta en una medición realizada por un grupo de expertos internos de la empresa o entidad, en la que se utiliza una encuesta para medir el riesgo de cada uno de los eventos identificados. Las encuestas son diseñadas para medir, en primer lugar, el riesgo inherente y después el riesgo residual. La aplicación de la encuesta es de manera independiente para luego consolidar los resultados de dichas mediciones.

La medición es desarrollada por personas que, ante todo, tienen un buen entendimiento de los clientes, productos, canales y jurisdicciones (entendidas como zonas en las cuales se hace negocios o actividades). El rol de experto es dar su opinión sobre los temas que se le van a preguntar, usando la información que tenga y su mejor criterio.

Para la agregación y desagregación de los riesgos por elemento, segmento, factor o riesgo asociado, se podrá utilizar una variable de repartición atada al volumen o frecuencia de las transacciones o actividades.

El método que se utiliza para la medición de la probabilidad y el impacto se fundamenta en una medición realizada por un grupo de expertos internos de la empresa, en la que se utiliza una encuesta para medir el riesgo de cada uno de los eventos identificados. Las encuestas son diseñadas para medir, en primer lugar, el riesgo inherente y después el riesgo residual. La aplicación de la encuesta es de manera independiente para luego consolidar los resultados de estas mediciones.

La medición es desarrollada por personas que, ante todo, tienen un buen entendimiento de los clientes, productos, canales y jurisdicciones de la empresa (entendidas como zonas en las cuales tenemos negocios). El rol del experto es dar su opinión sobre los temas que se le van a preguntar, usando la información que tenga y su mejor criterio.

Para la agregación y desagregación de los riesgos por elemento, segmento, factor o riesgo asociado, la empresa o entidad podrá utilizar una variable de repartición atada al volumen o frecuencia de las transacciones. 


\subsubsection{Procedimiento para medir el riesgo de lavado de activos y financiación del terrorismo}

Para la medición de los riesgos por medio del método de expertos se deben llevar a cabo las siguientes actividades:

Figura 2.13. Procedimiento para medir el riesgo de lavado de activos y financiación del terrorismo
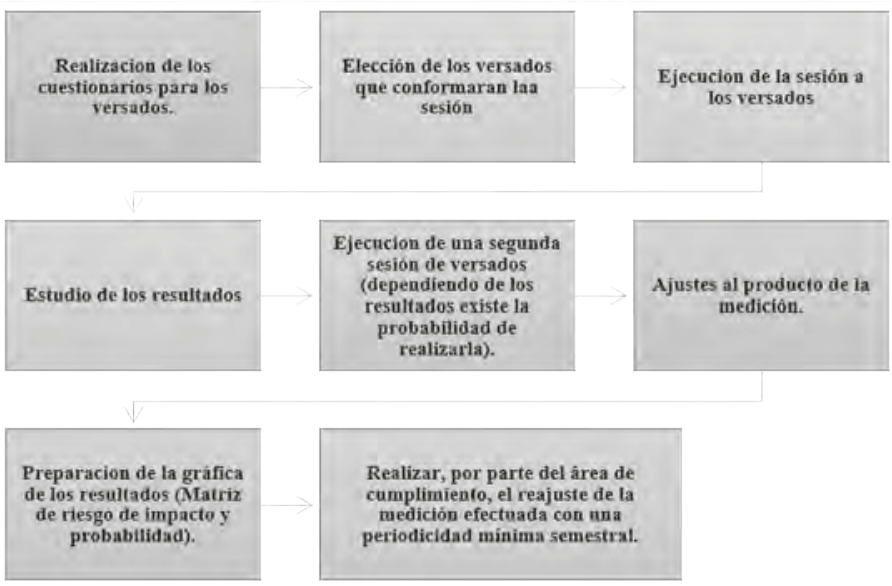

Realizar, por parte del área de cumplimiento, el reajuste de la ruedición efectuada con una periodicldad minima semestral.

Fuente: elaboración propia

\subsubsection{Control del riesgo de lavado de activos y financiación del terrorismo}

La metodología de tratamiento de los riesgos (control) que se recomienda es la contenida en el numeral 3.6. de la Norma Técnica Colombiana NTC 5254 de ICONTEC. 


\subsubsection{Procedimiento para definir las medidas de control}

Figura 2.14. Procedimiento para definir las medidas de control

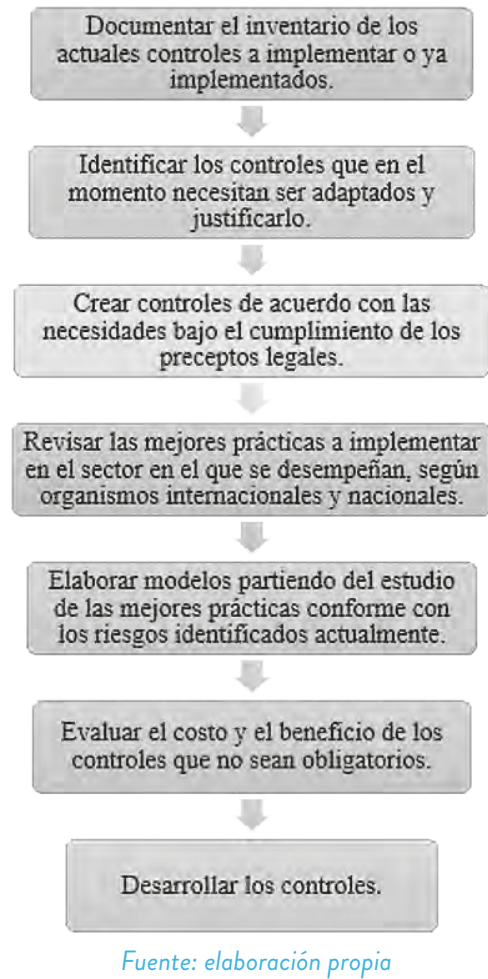

\subsubsection{Monitoreo del riesgo de lavado de activos y finan- ciación del terrorismo}

Para lograr un monitoreo oportuno y eficiente de operaciones susceptibles al LA/FT se recomienda tener en cuenta las acciones que se relacionan abajo: 
Figura 2.15. Monitoreo del riesgo de lavado de activos y financiación del terrorismo

El oficial de cumplimiento: debera efectuar una supervision continua del sistema con el propósito de valorar la eficacia de los controles.

El responsable(s) del proceso: deberá monitorear periódicamente los procedimientos y las tareas del proceso a su cargo, para con ello confirmar que no se hayan presentado nuevos eventos de riesgo y que los procedimientos y metodologias sigan siendo eficaces y apropiados.

Las áreas de control: la revisoria fiscal y la auditoria interna deben hacer seguimiento para localizar deficiencias, de acuerdo con las funciones asignadas por el SARL.AFT.

\section{Fuente: elaboración propia}

\subsubsection{Actividades para adelantar en la construcción de indicadores}

Para desarrollar el monitoreo del SARLAFT, los responsables en la empresa o entidad adelantarán las siguientes acciones:

* Para cumplir con la obligación de construir indicadores descriptivos y/o prospectivos que evidencien potenciales fuentes de riesgo de LA/FT, se habrá de establecer indicadores descriptivos como por ejemplo:

* Conocimiento del cliente: número de solicitudes de vinculación de clientes o de trabajadores o prestadores de servicios que fueron rechazadas.

Fórmula: operaciones no aprobadas / El total de solicitudes en el periodo determinado* 100 (se multiplica por 100 para que el resultado se exprese en porcentaje).

Periodicidad: esta se establece por la empresa conforme con las necesidades (mensual, semanal o diaria).

* Número de peticiones de vinculación con datos incompletos u otra característica (siempre deben ir identificadas por la causa del rechazo).

Fórmula: solicitudes con la información incompleta (causa del rechazo) / el total de solicitudes $* 100$

Periodicidad: mensual, semanal, diaria o la que se considere.

- Detección: porcentaje entre operaciones inusuales y reporte de operaciones sospechosas (ROS). 
Fórmula: número de operaciones inusuales / Número de reporte de operaciones sospechosas * 100

Periodicidad: trimestral.

* Número de intentos de vinculación de personas reportadas en listas de control (listas restrictivas como ONU, OFAC, noticias, procuraduría, policía, contraloría, registraduría, la lista interna que se tenga de clientes o terceros que generaron señales de alerta u operaciones sospechosas etc.).

Fórmula: número de intentos de vinculación de personas reportadas en listas de control.

Periodicidad: mensual.

* Porcentaje de atención a señales de alerta.

Fórmula: número de señales de alerta atendidas / Número total de señales de alerta generadas durante el mes *100

Periodicidad: mensual.

* Capacitación: Número de empleados o prestadores de servicios capacitados.

Fórmula: empleados o prestadores de servicios capacitados / número de empleados o prestadores de servicios *100.

Periodicidad: anual o semestral.

\subsubsection{Actividades por parte de cada responsable del} monitoreo

Figura 2.16. Actividades por parte de cada responsable del monitoreo

Revisión continúa por parte del oficial de cumpliniento de la actualización de las listas de control externas e interuas.

Selección aleatoria, por parte del oficial de cumplimiento, de información básica sobre clientes, tanto en la base de datos que maneja la enıpresa, con el objetivo de verificar la calidad de la información consignada en las bases de datos, como en los formularios de vinculación o ingreso.

Revision y adopción de las recomendaciones realizadas por el revisor fiscal en el reporte trimestral que realiza a la junta directiva en el proceso de evaluación del cumplimiento de las normas e instructivos sobre el SARLA/FI.

Revisión y adopción de las recomendaciones realizadas por el anditor interno en la evaluación anual de cada una de las etapas del SARLA/FT

Fuente: elaboración propia 


\subsubsection{Evaluación de controles}

Con la finalidad de realizar la calificación de los controles, el oficial de cumplimiento debe definir los atributos con los cuales ha de calificar los controles a implementar. De la misma forma, ha de establecer el peso de cada atributo para determinar la importancia de estos.

Una vez definido lo anterior, se califican los controles frente a los atributos definidos. Esta calificación puede realizarse por medio de la formulación de una encuesta a algunos expertos de la empresa, pertenecientes a las áreas de control y de cumplimiento cuando se hace por primera vez, y luego se adelanta de acuerdo con los resultados de la medición y análisis de las operaciones inusuales. Cada atributo es calificado por lo menos bajo cinco aspectos específicos definidos por el oficial de cumplimiento, conforme con las necesidades de la empresa o entidad.

\subsubsection{Periodicidad}

Los procesos de monitoreo, en cabeza del oficial de cumplimiento y el personal encargado de cada fase, deben tener una periodicidad mínima de seis meses.

Cada acción realizada en el proceso del monitoreo debe aparecer por escrito, así como los descubrimientos, y preferiblemente debe acompañar los informes a los órganos de control.

\subsubsection{Reportes}

Las constancias del desarrollo de cada actividad para el monitoreo del riesgo constituyen los reportes a que hace referencia el numeral 4.1.4. del título I, capítulo XI de la Circular externa 007 de 1996 (Circular básica jurídica), de la Superintendencia Financiera.

Para acatar la obligación de realizar reportes que evidencien la evolución de los riesgos, así como la eficiencia de los controles implementados, se deben realizar los siguientes reportes: 
Figura 2.17. Reportes Internos

\begin{abstract}
Reporte del oficial de cumplimiento en el que consten los resultados de las evaluaciones a la eficiencia y eficacia de los controles implementados.
\end{abstract}

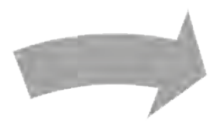

Un reporte del encargado del proceso sobre el cliente o el factor de riesgo. en el cual se le informe al oficial de cumplimiento que se encontró una falla dentro del proceso y a qué tipo de falla corresponde.

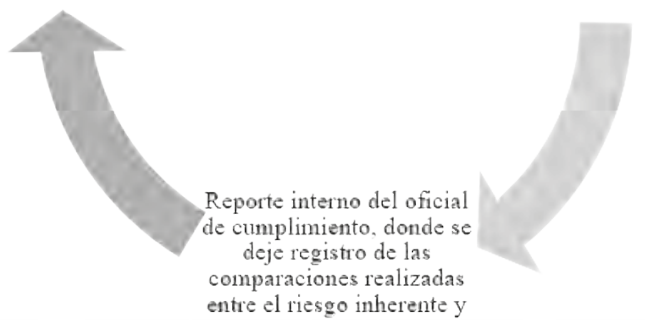

Fuente: elaboración propia

\subsubsection{Identificación de operaciones sospechosas}

El mercado se entiende como todo el grupo de operaciones que realizan los clientes y/o usuarios a partir de la utilización de los productos y servicios que ofrece.

Al adquirir un conocimiento del mercado y de los productos que lo integran, le permite a la empresa establecer cuáles son las características usuales de los agentes económicos que participan en él y las transacciones que desarrollan.

Para la segmentación de los mercados se deben diseñar perfiles de usuarios partiendo de la actividad económica y los valores de las operaciones realizadas asociados a sus ingresos previamente justificados, con lo que se podrá hacer una identificación de parámetros de monitoreo conforme con las características del mercado.

Para el conocimiento del mercado, la empresa o entidad ha de atender las siguientes características: 
Figura 2.18. Variables para la segmentación

\begin{tabular}{|c|}
\hline Monto \\
\hline Complejidad de las operaciones \\
\hline Regiones de alto riesgo de lavado de activos y financiamiento del terrorismo \\
\hline Clases de productos \\
\hline Actividad económica \\
\hline
\end{tabular}

Fuente: elaboración propia

Excepcionalmente, pueden presentarse operaciones de los clientes que difieren de las tendencias normales de los giros de sus negocios, por lo que se debe requerir soporte que justifiquen dicha situación, con lo que se podrá evidenciar si la señal de alerta pasa a ser una operación sospechosa objeto de reporte o no. Un caso común es el envío de giros por montos altos a beneficiarios con los que el cliente no tiene ningún vínculo aparente o jurisdicciones con alto riesgo de comisión de delitos, por lo que en caso de volverse reiterados y no justificados pasarán de ser señales de alerta a operaciones sospechosas.

\subsubsection{Identificación y análisis de operaciones inusuales}

Las operaciones inusuales son aquellas que contienen con las siguientes características mínimas: 


\subsubsection{Propuesta de metodología para la detección de operaciones}

No guardan relación con la actividad económica, o están fuera de los estándares establecidos por la empresa y, respecto de las cuales la empresa no ha encontrado explicación o justificación que se considere razonable.

\section{Fuente: elaboración propia}

* Inusuales: en la metodología para la detección es viable usar las señales de alerta recomendadas en el documento técnico de la UIAF-Colombia. La detección se toma como un control preventivo según el desarrollo de las etapas del SARLA/FT.

\subsubsection{Procedimiento para la detección de operaciones inusuales (seguimiento transaccional)}

* Todos los meses, la persona asignada en el área de cumplimiento debe comprobar las alertas detectadas por el sistema de la empresa o entidad. Estas se originan de acuerdo con los parámetros dados previamente en el sistema.

x Adicionalmente, es oportuno examinar las operaciones en efectivo, que individualmente o cuya sumatoria dé un valor superior al tope establecido en pesos por la empresa para un mes, con el fin de determinar posibles insuavidades.

x Luego de identificar las operaciones inusuales evidenciadas en el mes anterior, el signado por el área de cumplimiento deberá supervisar cada una de las operaciones teniendo en cuenta como variables las actividades económicas, ingresos, egresos, patrimonio y demás información del cliente con que cuenta la empresa. 
× Si lo considera oportuno, gestionará con el área encargada la justificación de aquellas actividades que dieron origen a la alerta, teniendo en cuenta que los soportes que se alleguen por parte del cliente deben ser coherentes con el monto y nivel de riesgo generado.

* Al finalizar el análisis, previamente documentado y archivado, la persona asignada por el área de cumplimiento deberá presentar sus conclusiones del caso al oficial de cumplimiento, quien decide si la operación está debidamente acreditada o no para su archivo o requiere de medidas especiales.

× Las operaciones inusuales que no sean justificadas debidamente serán llevadas al oficial de cumplimiento, quien decidirá si las operaciones cumplen o no con el carácter de sospechosas.

× Luego de ser identificadas las operaciones como sospechosas, el oficial de cumplimiento debe reportarlas a la UIAF.

En concordancia con la presencia de alertas generadas por el sistema, las investigaciones de operación inusual se pueden iniciar por los siguientes motivos:

Figura 2.20. Otros tipos de alerta

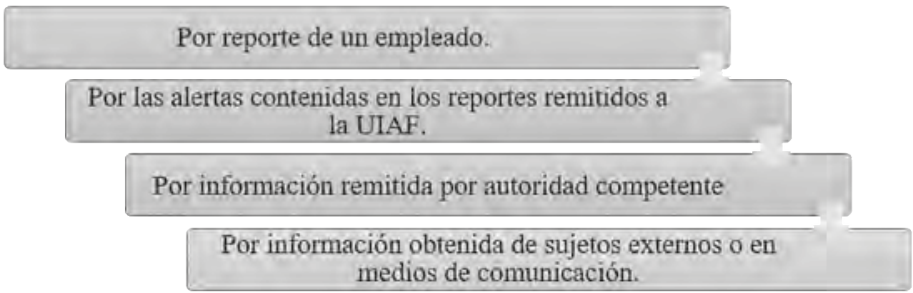

Fuente: elaboración propia

En el evento de detectar uno de los casos anteriormente mencionados, el analista de cumplimiento deberá iniciar la investigación.

\subsubsection{Determinación y reporte de operaciones sospecho- sas}

Una vez se identifican las operaciones inusuales, se da inicio al análisis de la información obtenida para corroborar si se trata o no de una operación sospechosa.

Quien decide si una operación pasa de ser inusual a sospechosa es el oficial de 
cumplimiento, ya que de estas últimas siempre se deberá hacer un reporte a la UIAF, denominado Reporte de operación sospechosa (ROS), y que debe emitirse desde el usuario de este. Es así que para definir la necesidad de emitir un $\mathrm{ROS}$ se recomienda atender las siguientes pautas:

× Verificar la existencia de antecedentes disciplinarios, penales o fiscales.

× Soportar el reporte con evidencias que demuestren que la operación es sospechosa.

× Hacer los análisis con absoluta objetividad e imparcialidad, solo basándose en las características de las señales de alerta de las operaciones, el perfil del cliente y el análisis de la segmentación.

No obstante, debe tenerse claro que el identificar una actividad como sospechosa no implica que esta sea efectivamente un delito, es decir, que se trata de una operación que no guarda relación con el perfil establecido para el cliente y se remite a la autoridad para que sea ella quien defina la existencia o no de un tipo penal.

\subsubsection{Reportes internos}

Corresponden a los que son de uso característico y único de la empresa.

\subsubsection{Reporte interno de transacciones inusuales $y / 0$ sospechosas}

En el evento que cualquier empleado en ejercicio de sus funciones revele cualquier operación que implique un hecho inusual, debe informarlo a su jefe inmediato, para que este de manera pronta lo ponga en conocimiento del oficial de cumplimiento, quien ha de iniciar los procesos de recolección de información y análisis para la identificación de operaciones sospechosas o reporte UIAF. Este reporte debe contener mínimamente la siguiente información: 

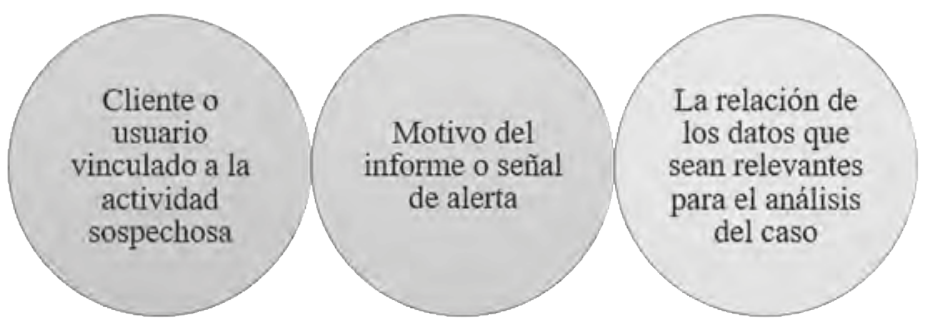

Fuente: elaboración propia

La información de las señales de alerta u operaciones inusuales detectadas por otras áreas pueden ser remitidas por medio de correo electrónico, informe escrito, llamada telefónica o de manera verbal.

\subsubsection{Reporte interno de la etapa de monitoreo}

Cada tres meses deben realizarse reportes internos de monitoreo en todas las áreas de la empresa a modo de autoevaluación, para construir los perfiles de riesgo residual (individuales y consolidados), verificar la evolución de cada uno de estos y así consolidar los perfiles de riesgo, los factores de riesgo y los riesgos asociados.

\subsubsection{Reportes externos}

La empresa o entidad debe enviar los reportes de acuerdo con las normas vigentes.

\subsubsection{Reporte de operaciones sospechosas (ROS)}

Los reportes de operaciones sospechosas solo pueden ser emitidos por el oficial de cumplimiento, quien deberá cargarlos con su usuario y contraseña en la plataforma institucional de la Unidad Especial de Información y Análisis Financiero del Ministerio de Hacienda y Crédito Público - UIAF (conforme a los parámetros del Anexo establecido por la Circular proferida por la Superintendencia Financiera de Colombia para este reporte).

Adicionalmente, se deben informar a la UIAF de todas aquellas operaciones que los clientes o usuarios quisieron realizar y que fueron rechazadas por ser inusuales, ya que esto puede facilitar la mitigación de riesgos futuros, siempre y cuando estas hayan sido detectadas siguiendo todos y cada uno de los parámetros definidos en el manual SARLAFT. 
Como no se requiere tener la certeza de que la operación sospechosa sea un acto delictivo ni tampoco tipificarlo, debido a que es una actividad de medio y no de resultado, sí es necesario que sea lo más objetivo posible y cuente con la mayor cantidad de elementos probatorios que demuestren su riesgo potencial, ya que se podrían estar remitiendo reportes no conducentes.

Si los procesos de monitoreo arrojan la inexistencia de actividades sospechosa por los clientes, usuarios o personal interno durante el mes, se deberá remitir un reporte negativo informando dicha situación dentro de los primeros 10 días del mes siguiente.

Todas las constancias de envío de reportes, al igual que los anexos de los casos, deben ser archivados en orden cronológico de manera física y digital, ya que la UIAF tiene un periodo largo de tiempo para revisar todos los informes remitidos y podrá requerir posteriormente soportes adicionales de los casos.

\subsubsection{Reporte de transacciones en efectivo}

La empresa o entidad, en cumplimiento del numeral $1^{\circ}$ del artículo 103 EOSF debe dejar constancia en formulario diseñado para el efecto de la información relativa a las transacciones en efectivo, cuyo valor sea igual o superior a las cuantías establecidas en el instructivo del anexo contenido en la Circular proferida por la Superintendencia Financiera de Colombia para este reporte, el cual debe enviarse a la UIAF dentro de los diez días calendario del mes siguiente al del corte.

\subsubsection{Reporte de clientes exonerados}

Cada empresa está en la libertad de determinar qué clientes deberán o no ser reportados por la realización de operaciones en efectivo, teniendo como base el monto individual y la suma de todos estos por mes, por lo que se recomienda poner topes mínimos y máximos de acuerdo con el perfil de cada cliente diario y por mes. En caso de exonerar clientes, deberá reportarlo mensualmente la compañía. 


\subsubsection{Reporte sobre operaciones de transferencia, remesa, compra y venta de divisas}

Cada tres meses debe elaborase un reporte a la UIAF de los clientes que realizaron operaciones de trasferencias internacionales, remesas y compra y venta de divisas. La remisión de este informe debe hacerse dentro de los diez días siguientes al corte del trimestre (siguiendo los parámetros del anexo establecido por la Circular proferida por la Superintendencia Financiera de Colombia para este reporte).

Las operaciones de recepción o envío de giros de divisas desde o hacia el exterior se reportan bajo la figura de transferencia internacional.

Las operaciones de derivados sobre divisas y las que se hacen con el Banco de la República o con intermediarios del mercado cambiario no deben ser reportadas, pues ellas cuentan con procesos de verificación en cabeza del Estado.

\subsubsection{Reporte sobre productos ofrecidos por las empresas vigiladas}

Todos los productos ofrecidos al público por una entidad vigilada por la Superfinanciera deben ser reportados a la UIAF por el oficial de cumplimiento dentro de los primeros diez días calendario del mes siguiente al corte del trimestre (siguiendo los parámetros del anexo establecido por la Circular proferida por la Superintendencia Financiera de Colombia para este reporte).

\subsubsection{Reporte de información sobre campañas políticas y partidos políticos}

Las empresas están en la libertad de decidir si abren o no cuentas, o manejan o no productos o servicios financieros con campañas políticas y partidos políticos. De hacerlo, deben reportarlo mensualmente. 